\title{
Endobronchial Ultrasound-Guided Transbronchial Needle Aspiration for Re-biopsy in Previously Treated Lung Cancer
}

\author{
Joohae Kim, MD ${ }^{1}$ \\ Hyo Jae Kang, MD \\ Sung Ho Moon, MD, PhD \\ Jong Mog Lee, MD' \\ Hyae Young Kim, MD, PhD' \\ Geon-Kook Lee, MD, PhD',2 \\ Jin Soo Lee, MD, PhD' \\ Bin Hwangbo, MD, PhD'
}

${ }^{1}$ Center for Lung Cancer, Research Institute and Hospital, National Cancer Center, Goyang, ${ }^{2}$ Department of Pathology, Research Institute and Hospital, National Cancer Center, Goyang, Korea
Correspondence: Bin Hwangbo, MD, PhD Center for Lung Cancer, Research Institute and Hospital, National Cancer Center, 323 Ilsan-ro, Ilsandong-gu, Goyang 10408, Korea Tel: 82-31-920-1718

Fax: 82-31-920-1275

E-mail: hbb@ncc.re.kr

Received January 12, 2019

Accepted March 12, 2019

Published Online March 15, 2019

\begin{abstract}
Purpose
Endobronchial ultrasound-guided transbronchial needle aspiration (EBUS-TBNA) is widely used for the diagnosis and staging of lung cancer. However, evidence of its usefulness for re-biopsy in treated lung cancer, especially according to the previous treatment, is limited. We evaluated the role of EBUS-TBNA for re-biopsy and its diagnostic values in patients with different treatment histories.
\end{abstract}

\section{Materials and Methods}

We reviewed the medical records of patients who underwent EBUS-TBNA for re-biopsy of suspicious recurrent or progressive lesions between January 2006 and December 2016 at the National Cancer Center in South Korea. Patients were categorized into three groups based on the previous treatment modalities: surgery, radiation, and palliation.

\section{Results}

Among the 367 patients (surgery, $n=192$; radiation, $n=40$; palliation, $n=135$ ) who underwent EBUS-TBNA for re-biopsy, the overall sensitivity, negative predictive value (NPV), and diagnostic accuracy of EBUS-TBNA in detecting malignancy were $95.6 \%, 82.7 \%$, and $96.3 \%$, respectively. The sensitivity was lower in the radiation group (83.3\%) when compared with the surgery $(95.7 \%, p=0.042)$ and palliation $(97.7 \%, p=0.012)$ groups. The NPV was lower in the palliation group (50.0\%) than in the surgery group $(88.5 \%, p=0.042)$. The sample adequacy of EBUS-TBNA specimens was lower in the radiation group (80.3\%) than in the surgery $(95.4 \%, p<0.001)$ or palliation $(97.8 \%, p<0.001)$ groups. EGFR mutation analysis was feasible in $94.6 \%$ of the 92 cases, in which mutation analysis was requested. There were no major complications. Minor complications were reported in 12 patients (3.3\%).

\section{Conclusion}

EBUS-TBNA showed high diagnostic values and high suitability for EGFR mutation analysis with regard to re-biopsy in patients with previously treated lung cancer. The sensitivity was lower in the radiation group and NPV was lower in the palliation group. The complication rate was low.

\section{Introduction}

When recurrent or progressive disease is suspected after the treatment of lung cancer, re-biopsy is frequently required to confirm disease status and perform molecular subtyping to guide appropriate treatment [1-3]. Re-biopsy should be considered to check for acquired mutations such as T790M,
Key words

Lung neoplasms, Endobronchial ultrasound, Biopsy especially after treatment with epidermal growth factor receptor tyrosine kinase inhibitors (EGFR-TKI) [1,3].

Re-biopsy was found to be technically difficult and accompanied by a higher rate of complications when compared to that of the first biopsy performed in a study of re-biopsy after EGFR-TKI treatment [4]. Trans-thoracic needle aspiration (TTNA) or trans-thoracic needle biopsy is widely used for the initial diagnosis of lung cancer and for re-biopsy $[5,6]$. 
However, the rate of complications associated with transthoracic procedures, such as pneumothorax, was found to be $14 \%-26 \%$ after re-biopsy in patients with previously treated advanced lung cancer $[6,7]$.

Endobronchial ultrasound-guided transbronchial needle aspiration (EBUS-TBNA) is a bronchoscopic sampling method that enables real time aspiration under ultrasound guidance. High diagnostic values of EBUS-TBNA were reported with regard to staging, diagnosis, and tissue acquisition for mutation analysis in lung cancer [8-10]. Moreover, EBUS-TBNA is a safe method, with a low rate of complications reported ranging between $0.15 \%$ and $1.23 \%[11,12]$.

The role of EBUS-TBNA for re-biopsy of lung cancer has been studied, showing high diagnostic values [2,13-19]. The sensitivities of EBUS-TBNA for re-biopsy were higher than $90 \%$ in most studies [2,13,15-19]. However, those studies enrolled a relatively small number of patients $(n=9-73)$ [2,13-19], most of whom had a history of surgery [2,13-16]. According to Han et al. [13] ( $n=42)$, the sensitivity of EBUSTBNA for diagnosing recurrent lesions was $94.3 \%$ in patients who underwent curative surgery. Anraku et al. [2] evaluated 44 patients, including 11 patients who underwent radiation or chemotherapy, and showed a high sensitivity $(93.1 \%)$ for re-biopsy using EBUS-TBNA. Evison et al. [16] analyzed 56 patients with previously treated lung cancer (surgery, $n=43$; radical radiation, $n=3$; chemoradiation, $n=6$; palliative chemotherapy, $n=4$ ) and suspected nodal metastases and reported that the overall sensitivity of EBUS-TBNA was $91.4 \%$. The role of EBUS-TBNA for re-biopsy in patients with progressive disease after chemotherapy or EGFR-TKI treatment were evaluated only in small case series ( $n=9$ [19], $n=18$ [18]) and showed a sensitivity of $100 \%$ in detecting malignancy [18,19].

Diagnostic values of EBUS-TBNA can vary according to the previous treatment modalities used. However, no studies have compared the role of EBUS-TBNA for re-biopsy in different clinical settings. In this large retrospective cohort study, we aimed to comprehensively evaluate the role of EBUS-TBNA in performing re-biopsies of suspicious recurrent or progressive lesions after lung cancer treatment. We compared the diagnostic values of EBUS-TBNA for re-biopsy in patients with different treatment histories. The suitability of re-biopsy samples obtained by EBUS-TBNA for EGFR mutation analysis was also evaluated.

\section{Materials and Methods}

\section{Patients and treatment groups}

This retrospective cohort study included patients who underwent EBUS-TBNA for the re-biopsy of suspected recurrent or progressive lesions after receiving lung cancer treatment between January 2006 and December 2016 at the National Cancer Center in South Korea. Patients were excluded if the EBUS-TBNA was performed for the purpose of re-staging the cancer after neo-adjuvant treatment or for clinical trial enrollment without evidence of disease progression.

The patients were divided into two groups-the curative group and palliation group. The curative group comprised two sub-groups: surgery group and radiation group. The surgery group included patients who underwent surgery \pm neo-adjuvant or adjuvant treatment with curative intent. The radiation group included patients who received radical radiation or chemotherapy+radiation with curative intent for the treatment of stage I-III non-small cell lung cancer or limited disease small cell lung carcinoma (SCLC). When radiation therapy was performed for the treatment of postoperative recurrent lesions with a curative purpose, the case was classified into the radiation group. The palliation group included patients who were treated with chemotherapy or target therapy for advanced lung cancer with palliative intent, irrespective of treatment modalities that were given prior to the palliative treatment, such as surgery or radiation.

\section{EBUS-TBNA procedure}

EBUS-TBNA was performed using an ultrasound bronchoscope and a dedicated ultrasound processor (convex probeEBUS, BF-UC260F-OL8 and EU-C2000, Olympus, Tokyo, Japan). Needle aspiration was performed with a 22-gauge needle (NA-201SX-4022, Olympus). The procedure was performed with the patient under conscious sedation (midazolam) and local anesthesia (lidocaine). One or more target lesions were selected at the bronchoscopists' discretion. When the main lesion that was suspicious for recurrence or progression was not accessible by EBUS-TBNA, the bronchoscopists selected targets that were still suspicious for malignancy and accessible by EBUS-TBNA as the second option. Thansbronchial needle aspirations were performed on target lesions. We allowed additional aspirations by endoscopic ultrasound with bronchoscope-guided fine needle aspiration (EUS-B-FNA) when we encountered difficulties with EBUSTBNA. The aspirate was placed into a solution of $10 \%$ neutral-buffered formalin. Cell-blocks were prepared and stained with hematoxylin and eosin. EGFR mutation analysis was performed with direct sequencing as described in the lit- 
erature [20] or peptide nucleic acid-mediated polymerase chain reaction clamping. A pathologist (G.K.L.) performed the cytopathologic and molecular examinations.

\section{Data collection}

We collected demographic and clinical data of the enrolled patients by reviewing their medical records. We examined baseline characteristics including age, sex, histologic diagnosis, staging of lung cancer at the time of initial diagnosis, sampling methods used for the initial histologic diagnosis, and time elapsed from initial diagnosis to re-biopsy. We also collected data regarding target lesions and details of the EBUS-TBNA procedures performed, such as the location and size of the targets, maximum standardized uptake value (mSUV) of the targets in patients with positron emission tomography-computed tomography (PET-CT) scans, number of aspirations per target during the EBUS-TBNA, number of tissue core samples obtained by EBUS-TBNA per target, procedure time, and complications associated with the procedure. When the main lesion suspicious for recurrence and progression was not targeted by EBUS-TBNA, we recoded the cases. Lymph node location was classified according to the International Association for the Study of Lung Cancer lymph node map [21]. We measured the short diameter of lymph nodes and the long diameter of non-nodal lesions on the axial chest computed tomography scans. The bronchoscopy ( \pm EUS-B) procedure time from the start to the end of the procedure was calculated; this included procedure time for a routine bronchoscopy performed before EBUS-TBNA. Major complications were defined as complications requiring admission or interventional procedures. Other complications were considered to be minor.

Treatment modalities were reviewed to categorize the enrolled patients as described above. In patients with previous radiation treatment, we reviewed whether or not the target lesion was located in the radiation field (delivered at least 20 Gy of radiation).

We reviewed the cytopathological findings of specimens. When EBUS-TBNA was performed repeatedly for re-biopsy, we analyzed the first result of EBUS-TBNA only. Cytopathological specimens were categorized as positive (presence of tumor cells), negative (lymphocytes, lymphoid tissue, inflammatory, or specific benign features), or inadequate (no or scanty cellular components, blood only, or cartilage or bronchial epithelial cells only). The histologic types of the malignancies diagnosed by re-biopsy were recorded. The clinicians' requests for EGFR mutation analysis, which was ordered before obtaining the EBUS-TBNA results, were reviewed. We recorded the major EGFR mutations (exon 19 deletion and exon 21 L858R mutation) and T790M mutation diagnosed by re-biopsy.

\section{Calculation of diagnostic values}

Diagnostic values, such as sensitivity, negative predictive value (NPV), and accuracy were calculated according to the standard definitions in a patient-based calculation. A patient with a positive result was considered to be a true positive case. In patients with a negative or inadequate result only, we considered the case to be a true negative when the target was confirmed to be benign by surgical biopsy or clinical follow-up (e.g., specific benign diseases such as sarcoidosis, tuberculosis, etc.) or if there was no evidence of progression by imaging studies for 6 months after the re-biopsy and without anti-cancer treatment for the target lesions. When the targets were confirmed to be malignant by other cytopathological methods or if they progressed within 6 months after re-biopsy, we considered the case to be a false-negative case. When a patient with a negative or inadequate result received a new anti-cancer treatment targeting the lesions or was not followed for 6 months after the re-biopsy, we considered the case as a not-applicable. Not-applicable cases were excluded from the calculations of prevalence, sensitivity, NPV, and accuracy.

Sample adequacy (proportion of positive or negative results) was determined using a lesion-based calculation. The suitability of specimens for EGFR mutation analysis (proportion of processed EGFR mutation analyses among requested cases) was calculated.

\section{Statistical analysis}

Baseline characteristics are summarized using descriptive statistics, such as the numbers and percentages, mean \pm standard deviation, or median (interquartile range [IQR]), as appropriate. Number of aspirated targets, number of aspirations per target prevalence, and diagnostic values (sensitivity, NPV, accuracy, and sample adequacy) were compared between groups by $t$ test, chi-square test or Fisher exact test, as appropriate. A p-value of $<0.05$ was considered statistically significant. All analyses were conducted using Stata Statistics ver. 14 (Stata Corp, College Station, TX).

\section{Ethical statement}

This study was approved by the National Cancer Center Institutional Review Board (IRB no. NCC2016-0257) and performed in accordance with the principles of the Declaration of Helsinki. The requirement for informed consent was waived. 
Table 1. Baseline characteristics of patients

\begin{tabular}{|c|c|c|c|c|}
\hline Characteristic & $\begin{array}{c}\text { All } \\
(n=367)\end{array}$ & $\begin{array}{l}\text { Surgery } \\
(n=192)\end{array}$ & $\begin{array}{l}\text { Radiation } \\
\qquad(n=40)\end{array}$ & $\begin{array}{l}\text { Palliation } \\
(n=135)\end{array}$ \\
\hline Age (yr) & $64.4 \pm 9.2$ & $65.9 \pm 8.0$ & $68.6 \pm 8.6$ & $61.3 \pm 9.9$ \\
\hline Male sex & $267(72.8)$ & $152(79.2)$ & $38(95)$ & $77(56.5)$ \\
\hline \multicolumn{5}{|l|}{ Histology of initial diagnosis } \\
\hline Adenocarcinoma & $199(54.2)$ & $82(42.7)$ & $12(30.0)$ & $105(78.3)$ \\
\hline Squamous cell lung cancer & $117(31.9)$ & $97(50.5)$ & $13(32.5)$ & $7(5.1)$ \\
\hline Large cell lung cancer & $5(1.4)$ & $2(1.0)$ & 0 & $3(2.2)$ \\
\hline Adenosquamous & $3(0.8)$ & $3(1.6)$ & 0 & 0 \\
\hline Sarcomatoid carcinoma & $4(1.1)$ & $3(1.6)$ & 0 & $1(0.7)$ \\
\hline Non-small cell lung cancer, others & $7(1.9)$ & $3(1.6)$ & $1(2.5)$ & $3(2.2)$ \\
\hline Small cell lung cancer & $26(7.1)$ & 0 & $13(32.5)$ & $13(9.4)$ \\
\hline Combined histology & $6(1.6)$ & $2(1.0)$ & $1(2.5)$ & $3(2.2)$ \\
\hline \multicolumn{5}{|l|}{ Stage at initial diagnosis } \\
\hline Non-small cell lung cancer & $341(93.0)$ & $192(100)$ & $26(65.0)$ & $123(91.1)$ \\
\hline Stage I & $105(28.6)$ & $91(47.4)$ & $11(27.5)$ & $3(2.2)$ \\
\hline Stage II & $59(16.1)$ & $51(26.6)$ & $5(12.5)$ & $3(2.2)$ \\
\hline Stage III & $70(19.1)$ & $48(25.0)$ & $10(25.0)$ & $12(8.9)$ \\
\hline Stage IV & $107(29.2)$ & $2(1.0)$ & 0 & $105(77.8)$ \\
\hline Small cell lung cancer & $26(7.1)$ & 0 & $14(35.0)$ & $12(8.9)$ \\
\hline Extensive disease & $11(3.0)$ & 0 & 0 & $11(8.1)$ \\
\hline Limited disease & $15(4.1)$ & 0 & $14(35.0)$ & $1(0.7)$ \\
\hline \multicolumn{5}{|l|}{ Sampling methods for initial diagnosis } \\
\hline TTNA or TTNB & $141(38.4)$ & $82(42.7)$ & $12(30.0)$ & $47(34.8)$ \\
\hline Bronchoscopic biopsya) & $125(34.1)$ & $71(36.9)$ & $18(45.0)$ & $36(26.7)$ \\
\hline EBUS-TBNA & 49 (13.4) & $9(4.7)$ & $8(20.0)$ & $32(23.7)$ \\
\hline Surgery & $32(8.7)$ & $27(14.0)$ & $1(2.5)$ & $4(3.0)$ \\
\hline Neck node or SCN sampling & $4(1.1)$ & 0 & $1(2.5)$ & $3(2.2)$ \\
\hline Pleural effusion cytology & $4(1.1)$ & 0 & 0 & $4(3.0)$ \\
\hline Others & $12(3.3)$ & $3(1.6)$ & 0 & $9(6.7)$ \\
\hline Time between initial and re-biopsy (mo) & $23.3 \pm 16.8$ & $24.3 \pm 16.4$ & $25.6 \pm 18.4$ & $21.1 \pm 16.8$ \\
\hline
\end{tabular}

Values are presented as mean \pm standard deviation or number (\%). TTNA, transthoracic needle aspiration; TTNB, transthoracic needle biopsy; EBUS-TBNA, endobronchial ultrasound-guided transbronchial needle aspiration; SCN, supra-clavicular lymph node. ${ }^{\text {a) }}$ Endobronchial or transbronchial biopsy.

\section{Results}

\section{Patient characteristics, target lesions, and EBUS-TBNA procedures}

Of the 5,710 patients who underwent EBUS between 2006 and 2016 at the National Cancer Center, EBUS-TBNA was performed for re-biopsy in 367 lung cancer patients. Baseline characteristics of all the patients and their classification into the different treatment groups are presented in Table 1. The mean patient age was 64.4 years, and the proportion of male patients was $72.8 \%$. Although we did not examine the differences in initial histology and staging between groups by sta- tistical analysis, we could observe these differences, such as the proportion of SCLC and advanced disease between groups that are relevant to treatment options. For the initial histologic diagnosis, transthoracic sampling methods were used in $38.4 \%$ of patients, and bronchoscopic methods were used in $47.4 \%$ of patients. The mean elapsed time from the initial diagnosis to the EBUS re-biopsy was 23.3 months.

In $88.8 \%$ ( $n=326)$ of patients, the main lesion that was suspicious for recurrence or progression was targeted. In $11.2 \%$ $(n=41)$ of cases, the main targets were not or could not be accessed, most of which were in the palliation group $(n=32)$. In 33 patients, EBUS-TBNA procedures were repeated more than twice, in which case we evaluated only the result of the first biopsy for calculation of diagnostic values. 
Table 2. Characteristics of target lesions and EBUS-TBNA procedures

\begin{tabular}{|cc}
\hline Characteristic & Value \\
\hline Location of target lesions & 544 \\
\hline Mediastinal nodes & $302(55.5)$ \\
\hline Hilar/Interlobar or peripheral nodes & $126(23.2)$ \\
\hline Bronchial stump or peribronchial soft tissue & $19(3.5)$ \\
\hline Lung parenchymal lesion & $96(17.6)$ \\
\hline Pleural lesion & $1(0.2)$ \\
\hline Target lesions per patients & \\
\hline Lymph nodes only & $256(69.8)$ \\
\hline Lymph nodes and others ${ }^{\text {a) }}$ & $81(22.1)$ \\
\hline Parenchymal/Stump/Peribronchial/ & $30(8.2)$ \\
\hline Pleural lesion only & \\
\hline Size of target on axial CT images (mm) & \\
\hline Lymph nodes & $13.0 \pm 5.3$ \\
\hline Non-nodal lesions & $39.3 \pm 21.1$ \\
\hline PET-CT, patients & $286(77.9)$ \\
\hline PET SUV max & $7.8 \pm 4.2$ \\
\hline Procedure & \\
\hline No. of needle aspiration/Target & $3.5 \pm 1.4$ \\
\hline No. of tissue core samples/Target & $2.8 \pm 1.3$ \\
\hline Procedure time, min & $20.9 \pm 8.4$ \\
\hline Major complications & 0 \\
\hline Minor complications & $12(3.3)$ \\
\hline Desaturation during procedure & $6(1.6)$ \\
\hline Transient fever & $3(0.8)$ \\
\hline Minor bleeding & $2(0.5)$ \\
\hline Transient chest pain & $1(0.3)$ \\
\hline Values & \\
\hline
\end{tabular}

Values are presented as number $(\%)$ or mean \pm standard deviation. EBUS-TBNA, endobronchial ultrasound-guided transbronchial needle aspiration; $\mathrm{CT}$, computed tomography; PET-CT, positron emission tomography-computed tomography; $\mathrm{SUV}_{\max }$, maximum standardized uptake values. ${ }^{\text {a) }}$ Others: parenchymal, stump, or peribronchial lesions.

Characteristics of target lesions and details of the procedures are listed in Table 2 and S1 Table. Among the 367 patients, 544 targets were sampled (average, 1.48 lesions/patient). In 19 patients (5.2\%), EUS-B-FNA was added following EBUS-TBNA on 23 lesions ( $4 \mathrm{~L}, \mathrm{n}=11 ; 7, \mathrm{n}=6 ; 2 \mathrm{~L}, \mathrm{n}=2 ; 1 \mathrm{R}$, $\mathrm{n}=2$; $2 \mathrm{R}, \mathrm{n}=1 ; 10 \mathrm{~L}, \mathrm{n}=1)$. Lymph nodes were the most common targets $(78.7 \%)$, with the remaining targets comprising bronchial stump/peribronchial soft tissue $(3.5 \%)$, lung parenchymal lesions $(17.6 \%)$, and pleural lesions $(0.2 \%)$. The mean sizes of the aspirated lymph nodes and non-nodal lesions were $13.0 \mathrm{~mm}$ (range, 5.1 to $41.0 \mathrm{~mm}$ ) and $39.3 \mathrm{~mm}$ (range, 9.8 to $108.2 \mathrm{~mm}$ ), respectively. The mean mSUV of the targets was 7.8 in 286 patients who underwent PET-CT. The number of needle aspirations and tissue cores per target were 3.5 and
2.8 , respectively. The average time for the procedure was 20.9 minutes (data available in 277 patients). There were no major complications, and only minor complications were observed in $3.3 \%$ of patients $(n=12)$ (Table 2$)$.

\section{Diagnostic values of EBUS-TBNA and subgroup analysis according to treatment}

Diagnostic values of EBUS-TBNA are presented in Table 3. In patient-based assessment, we did not analyze the result of EUS-B-FNA separately. Malignant cells were detected in 366 lesions of 280 patients $(76.3 \%)$. In 87 patients (23.7\%) with negative or inadequate results only, the median follow-up duration was 28.2 months (IQR, 17.2 to 52.2 months). We had 62 true-negative cases including one patient with sarcoidosis and 13 false-negative cases. Among the 13 false-negative cases, imaging follow-up showed disease progression in 12 patients (confirmed by repeated EBUS-TBNA, $n=2$ ). In one patient, malignancy was confirmed by surgical resection. Twelve patients (3.3\%) were classified as not applicable (follow-up $<6$ months, $n=9$; treatment given, $n=3$ ). After excluding these 12 patients, the prevalence of malignancy in this re-biopsy group was determined to be $82.5 \%$, and the sensitivity, NPV, and accuracy of EBUS-TBNA for re-biopsy was $95.6 \%, 82.7 \%$, and $96.3 \%$, respectively.

Subgroup analyses according to previous treatments are presented in Table 3 (curative group, $\mathrm{n}=232$ [surgery group, $\mathrm{n}=192$; radiation group, $\mathrm{n}=40$ ]; palliation group, $\mathrm{n}=135$ ). In comparison with the curative and palliation groups, the prevalence was significantly lower in the curative group (73.9\% vs. $97.7 \%, \mathrm{p}<0.001)$. The average number of aspirated targets was higher in the curative group $(1.6 \pm 0.8)$ than in the palliation group $(1.3 \pm 0.6, \mathrm{p}=0.002)$ and number of aspirations per target was higher in the palliation group $(4.3 \pm 1.5)$ than in the curative group $(3.1 \pm 1.1, \mathrm{p}<0.001)$ (data not presented in the Table).

We compared the diagnostic values of EBUS-TBNA between the surgery, radiation, and palliation groups. The sensitivity was lower in the radiation group $(83.3 \%)$ when compared with the surgery $(95.7 \%, \mathrm{p}=0.042)$ and palliation $(97.7 \%, \mathrm{p}=0.012)$ groups. The sensitivity was not different between the surgery and palliation groups $(95.7 \%$ vs. $97.7 \%$, $\mathrm{p}=0.503)$. The NPV was lower in the palliation group (50.0\%) than in the surgery group $(88.5 \%, \mathrm{p}=0.042)$. The NPV did not differ between the radiation and surgery groups $(76.5 \%$ vs. $88.5 \%, \mathrm{p}=0.254$ ) or radiation and palliation groups ( $76.5 \%$ vs. $50.0 \%, \mathrm{p}=0.319)$. The accuracy was lower in the radiation group $(89.2 \%)$ compared with the palliation group $(97.7 \%$, $\mathrm{p}=0.041$ ), although we could not observe a statistical difference in comparison with the surgery group $(96.8 \%, \mathrm{p}=0.065)$. The accuracy was not different between the surgery and palliation groups $(96.8 \%$ vs. $97.7 \%, \mathrm{p}=0.739)$. The sample ade- 


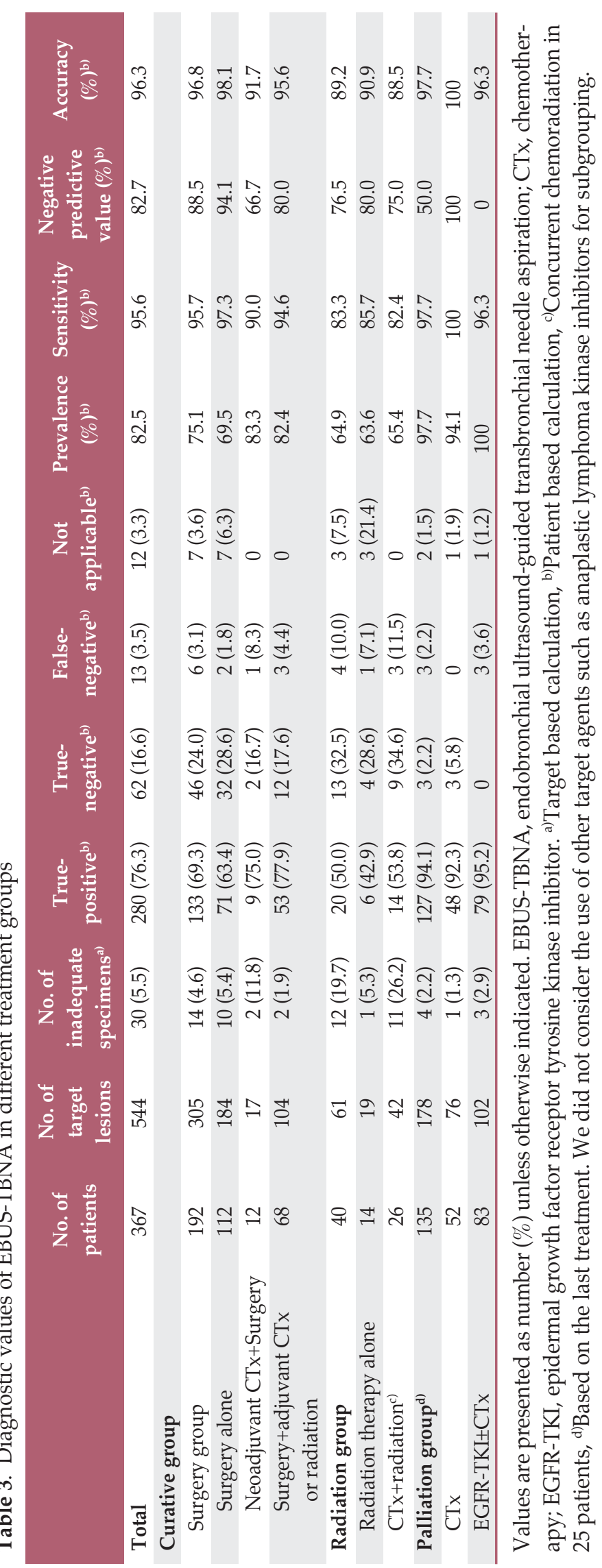




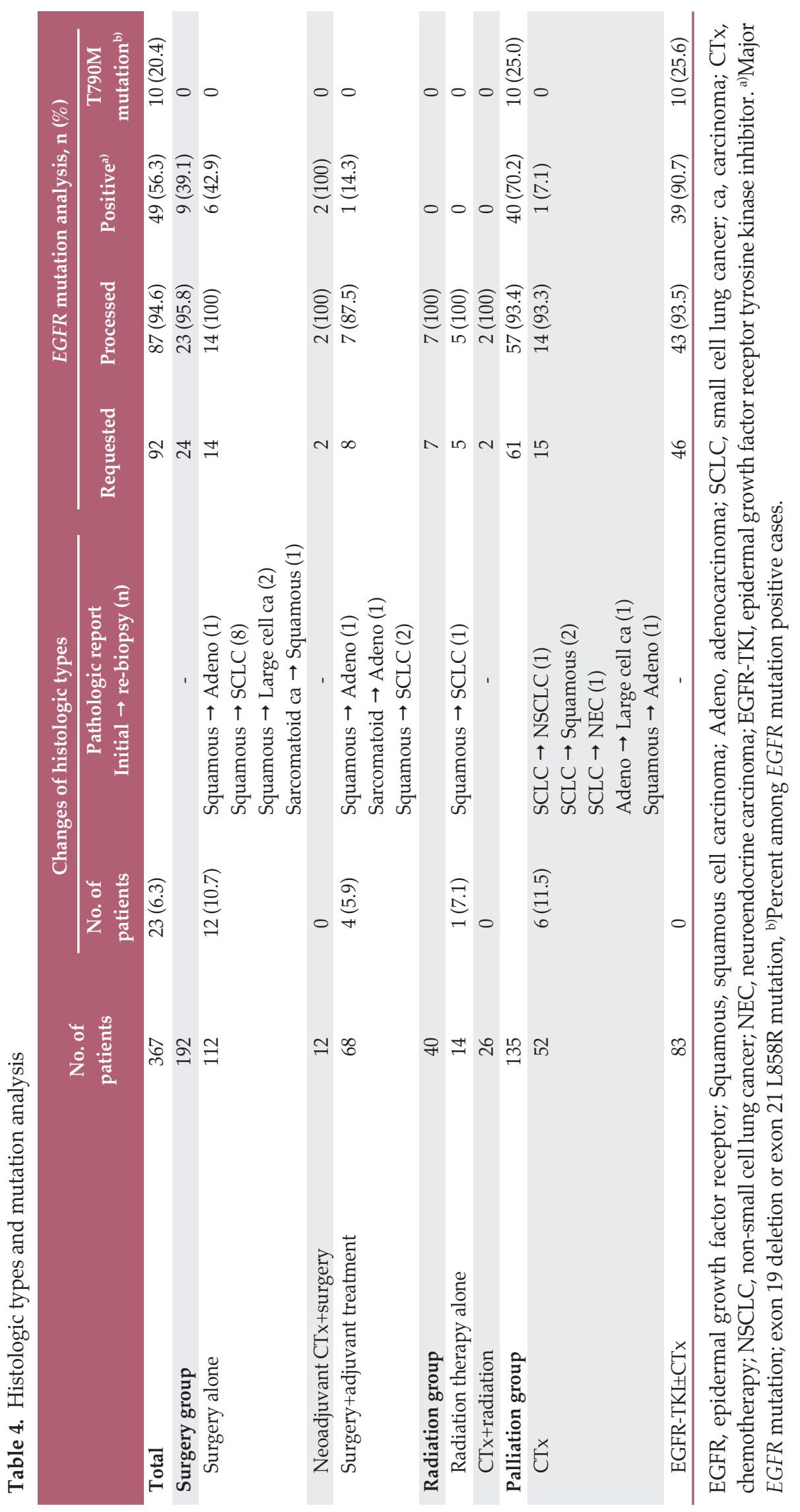



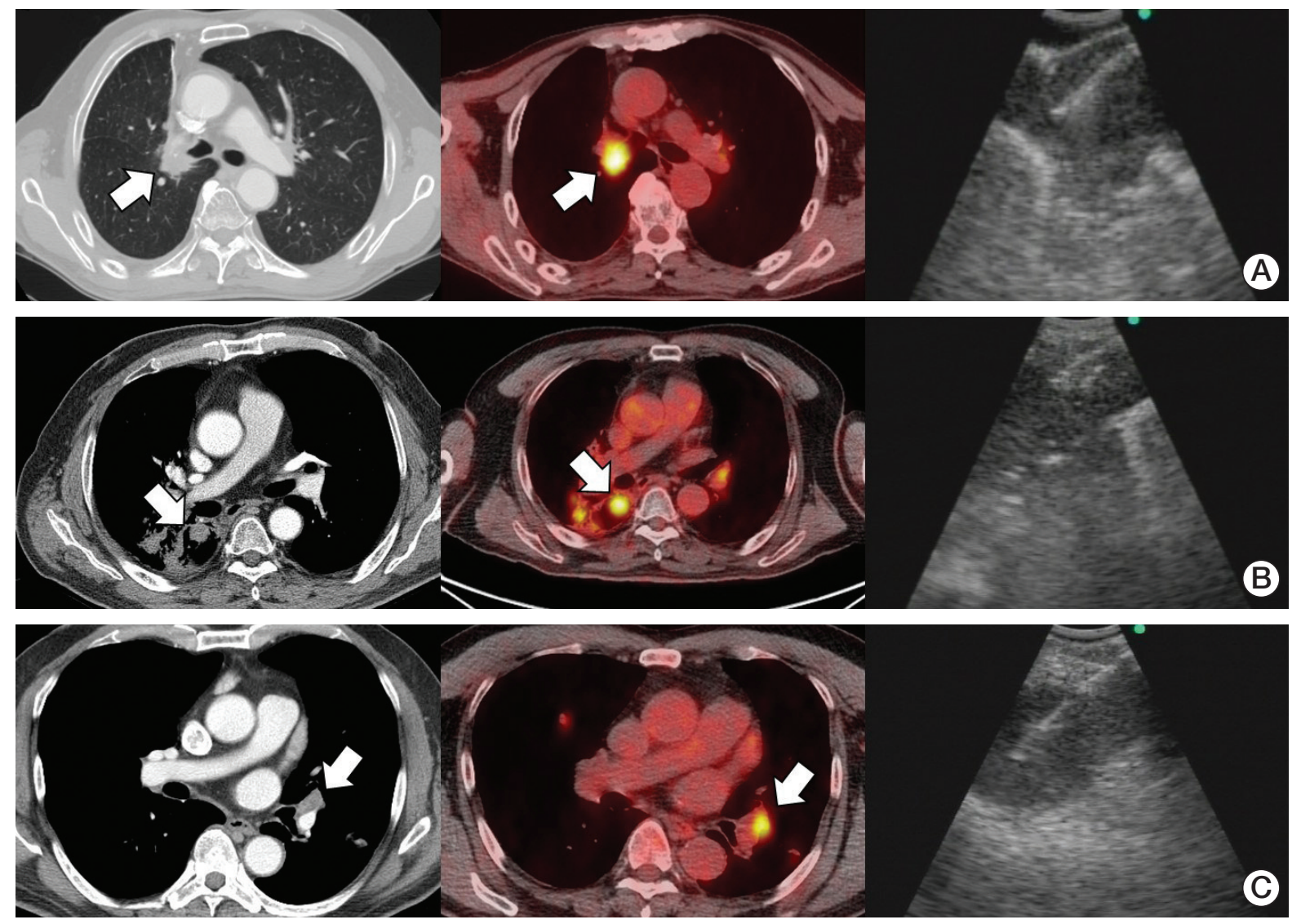

Fig. 1. (A) A 75-year-old man with adenocarcinoma, initial stage IB. Recurrence at the stump site was suspected one year after right upper lobectomy. Endobronchial ultrasound-guided transbronchial needle aspiration (EBUS-TBNA) confirmed recurrence. (B) A 65-year-old man with small cell lung cancer, initial stage of limited disease. Nodules in the radiation therapy field were observed 1.5 years after concurrent chemoradiation therapy. EBUS-TBNA diagnosed small cell lung cancer. (C) A 65-year-old man with adenocarcinoma, epidermal growth factor receptor (EGFR) mutation (L858R), initial stage IV. Cancer progression was suspected after treatment with gefitinib and erlotinib. EBUS-TBNA for re-biopsy detected EGFR T790M mutation.

quacy of the specimens was lower in the radiation group $(80.3 \%)$ than in the surgery group $(95.4 \%, \mathrm{p}<0.001)$ or palliation group $(97.8 \%, \mathrm{p}<0.001)$. We did not observe any difference in sample adequacies between the surgery and palliation groups ( $95.4 \%$ vs. $97.8 \%, \mathrm{p}=0.222$ ) (Table 3 ).

In the surgical group, EBUS-TBNA was performed in stump sites in 14 cases $(7.3 \%)$, and eight cases $(57.1 \%)$ were diagnosed as being malignant. The sensitivity and accuracy of EBUS-TBNA in stump re-biopsy were both $100 \%$. In the radiation group, targets were located within the radiation field in 28 cases, whereas the others were located outside of the field only $(\mathrm{n}=12)$. In the radiation group, targets were located within radiation field in the all false-negative cases $(\mathrm{n}=4)$.

\section{Histologic types, mutation analysis, and treatment choice}

Among the 367 enrolled patients, $23(6.3 \%)$ had a different histologic type than the initial diagnosis. Among them, 17 were in the curative group, and the lesions were considered to be new second primary tumors. In the remaining six patients, re-biopsy was performed in the palliation group; all six patients were judged to have second primary tumors and SCLC transformation was not observed in the palliation group (Table 4).

At the time of re-biopsy, EGFR analysis was requested in $32.9 \%$ of 280 patients with positive EBUS-TBNA results $(\mathrm{n}=92)$. Among the 92 patients, EGFR mutation analysis was not processed in five cases due to sample inadequacy. Therefore, the suitability for EGFR mutation analysis was $94.6 \%$ 
( $\mathrm{n}=87)$. Major EGFR mutations were detected in 49 patients $(56.3 \%)$. Among the 49 patients, $39(79.6 \%)$ had previously used TKI and $10(25.6 \%)$ were confirmed to have the T790M mutation.

The treatment plan was determined based on the results of the re-biopsy. Among the 280 cases with confirmed recurrent or progressive disease, most patients $(\mathrm{n}=244,87.1 \%)$ were switched to other treatment methods or new regimens of chemotherapy. No treatment decision was rendered in 36 patients $(12.9 \%)$, which was a result of poor performance status or the patient's decision to stop further treatment.

\section{Discussion}

In this retrospective study, we evaluated a relatively larger number of EBUS-TBNA cases for re-biopsy compared to previous studies [2,13-19]. We observed high diagnostic values of EBUS-TBNA in diagnosing disease recurrence or progression. The sensitivity, NPV, and accuracy of EBUS-TBNA for re-biopsy were $95.6 \%, 82.7 \%$, and $96.3 \%$, respectively. No major complications in the procedures were observed. There were only minor complications in 12 patients (3.3\%). Other studies also showed high sensitivities of EBUS-TBNA for rebiopsy in postoperative groups $(80.9 \%-100 \%)$ [13-15], mixed treatment groups (91.4\%-93.1\%) [2,16], and palliative groups (100\% in two studies) [18,19], although their sample numbers were smaller than ours. The results of our study showed that EBUS-TBNA was effective and safe for performing re-biopsies of lung cancer.

The strength of our study lies within the subgroup analysis according to treatment. Previous studies were more focused on the role of EBUS-TBNA in postoperative recurrence [13-15], and re-biopsy in a palliative setting was only analyzed in a small case series $[18,19]$. Moreover, the role of EBUS-TBNA for re-biopsy after radiation therapy was not separately investigated. In this study, we divided patients into three groups according to the treatment they had received: surgery, radiation, and palliation. The surgery group and radiation group belong to curative group and the palliation group included patients with advanced lung cancer treated with palliative intent. Essentially, the palliation group had a higher prevalence of malignancy, which is relevant to clinical settings.

In the surgery group (Fig. 1A), we observed high diagnostic values of EBUS-TBNA (sensitivity, 95.7\%; NPV, 88.5\%; and accuracy, 96.8\%). After surgical resection of the lung, anatomical changes, such as obstruction, narrowing, or distortion, inevitably occur $[22,23]$. However, our study shows that EBUS-TBNA can be effectively used for postoperative recurrence when the target is accessible by EBUS. In this study, EBUS-TBNA was performed in the stump site in 14 cases $(7.3 \%)$, and the sensitivity of EBUS-TBNA in the stump re-biopsy was $100 \%$. Diagnosing lesions around the surgical stump can be technically difficult due to fibrosis and the surgical materials. However, our results show that EBUS-TBNA is effective for stump biopsy.

In the radiation group (Fig. 1B), the sensitivity, NPV, and diagnostic accuracy of EBUS-TBNA were $83.3 \%, 76.5 \%$, and $89.2 \%$, respectively. The sensitivity was lower in the radiation group when compared to other groups. Additionally, the accuracy was lower compared to that of the palliation group. Radiation therapy induces inflammation or fibrosis and can cause aggressive and irreversible structural changes $[24,25]$. We believe that these diagnostic values in the radiation group are affected by radiation changes, such as fibrosis, that make an EBUS-TBNA procedure difficult. We observed that sample adequacy was also significantly lower in the radiation group $(80.3 \%)$, which further supports our explanation. Moreover, all false negative results were observed in cases with target lesions in the radiation field, which also suggests the technical limitations of EBUS-TBNA in the radiation field. We think the EBUS-TBNA results obtained in the radiation field are not sufficiently acceptable, and thus, careful clinical follow-up is needed.

In the palliation group (Fig. 1C), the sensitivity, NPV, and diagnostic accuracy of EBUS-TBNA were $97.7 \%, 50.0 \%$, and $97.7 \%$, respectively. To our knowledge, this is the largest case series regarding the use of EBUS-TBNA for re-biopsy after palliative treatment. Chemotherapy can cause structural lung damages [26] and may result in a lower diagnostic yield of EBUS-TBNA in the neo-adjuvant setting $[27,28]$. However, our results show that EBUS-TBNA is a highly sensitive and accurate method for re-biopsy of progressive lesions in the palliative setting. According to studies of TTNA, the diagnostic yield of re-biopsy in advanced lung cancer was about 82\%-85\% [6,7]. Although, direct comparisons between EBUSTBNA and TTNA is difficult to perform in cases of re-biopsy, the high complication rate associated with TTNA $[6,7]$ suggests that EBUS-TBNA is the preferable method for re-biopsy of progressive lesions when the target is accessible by EBUS. In the palliation group, we targeted lesions other than the main lesions in 32 patients, which suggest that EBUS-TBNA can be performed when other methods are difficult or not feasible. However, we observed a relatively low NPV for EBUS-TBNA in the palliation group. In this study, number of targets were lower (mean, $1.3 \mathrm{vs.} 1.6$ ) and number of aspirations per target was higher (mean, 4.3 vs. 3.1 ) in the palliation group compared with curative group; which may be related with the purposes of procedures such as confirming the extent of recurrence in the curative group and tissue acquisition in the palliation group. The number of needle 
passes required for re-biopsy for progressive disease was not determined. One study suggested four aspirations for molecular analysis [29]. Considering the number of aspirations in the palliation group of this study, our low NPV may not be related to the inadequacy of the EBUS-TBNA procedures. We believe this low NPV is related to the high prevalence of malignancy in advanced disease stages. Thus, the effectiveness of EBUS-TBNA cannot be underestimated based on the low NPV and target selection seems to be important.

In this study, the results of the EBUS-TBNA re-biopsies altered the treatment management in $87.1 \%$ of patients with positive results. In $6.3 \%$ of patients, we observed different histologic types than that of the initial diagnosis. Anraku et al. [2] reported new primary lung cancers in $16 \%$ (7/44) of postoperative patients who underwent EBUS-TBNA for re-biopsy. Diagnosing second primary cancers is an important role of EBUS-TBNA, especially in the curative setting. In the palliative setting, we observed several cases exhibiting different histology than that of the initial diagnosis, which were suspicious for second primary cancers. However, we did not observe SCLC transformation which was reported to occur in about $3 \%-14 \%$ of patients with EGFR resistance $[30,31]$.

In the present study, the suitability $(94.6 \%)$ for EGFR mutation analysis with re-biopsy samples obtained by EBUSTBNA was high. According to Navani et al. [10], EGFR mutation analysis was possible in $90 \%$ of patients in whom it was requested. However, they did not perform EBUSTBNA for re-biopsy. Therefore, our result is meaningful because EGFR mutation analysis is the main reason for re-biopsy in patients treated with EGFR-TKIs. We observed acquired T790M mutations in $25.6 \%$ of patients who were treated with EGFR-TKI, which is lower than the proportions reported in the literature $(50 \%-60 \%)[4,32,33]$. This may be because we used EBUS-TBNA mainly in the lymph nodes. In some patients, EBUS-TBNA was not performed in the main suspicious lesion due to inaccessibility or technical difficulty. The discordance in EGFR mutation results was shown between the primary tumor and the metastatic nodes $[20,34]$. Spatiotemporal T790M heterogeneity was also observed in a study, which performed re-biopsies at multiple sites after TKI treatment [35]. Selection bias may have affected the result, as we did not perform EGFR mutation analysis in all patients with progressive disease after EGFR-TKI treatment.

This study has several limitations. First, we could not surgically confirm negative or inadequate results. Therefore, true- or false-negative cases judged by clinical follow-up may lead to false interpretations. However, previous studies regarding re-biopsy in lung cancer patients calculated diagnostic values using similar definitions of positivity or negativity $[13,15,16]$. Second, due to the retrospective nature of this study, the targets were selected at the bronchoscopists' discretion. We could not provide specific guidelines for the selection of targets, such as that based on size or PET uptake. The accessibility by EBUS-TBNA according to the location of targets was also determined by bronchoscopists. The relatively small number of patients who underwent EGFR mutation analysis is another limitation. Finally, we added EUSB-FNA, as described in the literature [36], following EBUSTBNA in a small proportion of patients. We did not analyze the result of EUS-B-FNA separately, because we did not provide specific indications for adding EUS-B-FNA in this retrospective study. More studies are required to further investigate the role of EUS-B-FNA in re-biopsy.

In summary, EBUS-TBNA showed high diagnostic values for the re-biopsy of suspicious recurrent or progressive lesions in previously treated lung cancer. It is found to be a safe method with a low complication rate. In the radiation group, this method exhibited a relatively lower sensitivity, and the palliation group had a lower NPV. The suitability for EGFR mutation analysis was high in the patients for whom it was requested. Additionally, the use of EBUS-TBNA for re-biopsy largely affected the treatment management. Therefore, EBUS-TBNA should be considered for re-biopsy in lung cancer, although the results can be affected by previous treatment.

\section{Electronic Supplementary Material}

Supplementary materials are available at Cancer Research and Treatment website (https: // www.e-crt.org).

\section{Conflicts of Interest}

Conflict of interest relevant to this article was not reported.

\section{Acknowledgments}

This work was supported by National Cancer Center Grant 1610240. 


\section{References}

1. Jekunen AP. Role of rebiopsy in relapsed non-small cell lung cancer for directing oncology treatments. J Oncol. 2015;2015: 809835.

2. Anraku M, Pierre AF, Nakajima T, de Perrot M, Darling GE, Waddell TK, et al. Endobronchial ultrasound-guided transbronchial needle aspiration in the management of previously treated lung cancer. Ann Thorac Surg. 2011;92:251-5.

3. Novello S, Barlesi F, Califano R, Cufer T, Ekman S, Levra MG, et al. Metastatic non-small-cell lung cancer: ESMO Clinical Practice Guidelines for diagnosis, treatment and follow-up. Ann Oncol. 2016;27:v1-27.

4. Nosaki K, Satouchi M, Kurata T, Yoshida T, Okamoto I, Katakami N, et al. Re-biopsy status among non-small cell lung cancer patients in Japan: a retrospective study. Lung Cancer. 2016;101:1-8.

5. Rivera MP, Mehta AC, Wahidi MM. Establishing the diagnosis of lung cancer: Diagnosis and management of lung cancer, 3rd ed: American College of Chest Physicians evidence-based clinical practice guidelines. Chest. 2013;143(5 Suppl):e142S-65S.

6. Yoon HJ, Lee HY, Lee KS, Choi YL, Ahn MJ, Park K, et al. Repeat biopsy for mutational analysis of non-small cell lung cancers resistant to previous chemotherapy: adequacy and complications. Radiology. 2012;265:939-48.

7. Tokaca N, Barth S, O'Brien M, Bhosle J, Fotiadis N, Wotherspoon A, et al. Molecular adequacy of image-guided rebiopsies for molecular retesting in advanced non-small cell lung cancer: a single-center experience. J Thorac Oncol. 2018;13:6372.

8. Silvestri GA, Gonzalez AV, Jantz MA, Margolis ML, Gould MK, Tanoue LT, et al. Methods for staging non-small cell lung cancer: Diagnosis and management of lung cancer, 3rd ed: American College of Chest Physicians evidence-based clinical practice guidelines. Chest. 2013;143(5 Suppl):e211S-50S.

9. Lee JE, Kim HY, Lim KY, Lee SH, Lee GK, Lee HS, et al. Endobronchial ultrasound-guided transbronchial needle aspiration in the diagnosis of lung cancer. Lung Cancer. 2010;70:51-6.

10. Navani N, Brown JM, Nankivell M, Woolhouse I, Harrison $\mathrm{RN}$, Jeebun V, et al. Suitability of endobronchial ultrasoundguided transbronchial needle aspiration specimens for subtyping and genotyping of non-small cell lung cancer: a multicenter study of 774 patients. Am J Respir Crit Care Med. 2012;185:1316-22.

11. Gu P, Zhao YZ, Jiang LY, Zhang W, Xin Y, Han BH. Endobronchial ultrasound-guided transbronchial needle aspiration for staging of lung cancer: a systematic review and metaanalysis. Eur J Cancer. 2009;45:1389-96.

12. Asano F, Aoe M, Ohsaki Y, Okada Y, Sasada S, Sato S, et al. Complications associated with endobronchial ultrasoundguided transbronchial needle aspiration: a nationwide survey by the Japan Society for Respiratory Endoscopy. Respir Res. 2013;14:50

13. Han SG, Yoo H, Jhun BW, Park HY, Suh GY, Chung MP, et al. The role of endobronchial ultrasound-guided transbronchial needle aspiration in the diagnosis of recurrent non-small cell lung cancer after surgery. Intern Med. 2013;52:1875-81.

14. Sanz-Santos J, Serra P, Andreo F, Torky M, Centeno C, Moran $\mathrm{T}$, et al. Transbronchial and transesophageal fine-needle aspiration using a single ultrasound bronchoscope in the diagnosis of locoregional recurrence of surgically-treated lung cancer. BMC Pulm Med. 2017;17:46.

15. Yamamoto T, Sakairi Y, Nakajima T, Suzuki H, Tagawa T, Iwata $\mathrm{T}$, et al. Comparison between endobronchial ultrasound-guided transbronchial needle aspiration and 18F-fluorodeoxyglucose positron emission tomography in the diagnosis of postoperative nodal recurrence in patients with lung cancer. Eur J Cardiothorac Surg. 2015;47:234-8.

16. Evison M, Crosbie PA, Califano R, Summers Y, Martin J, Barber PV, et al. Can EBUS-TBNA provide an accurate diagnosis in patients found to have enlarged or FDG-avid lymph nodes during surveillance of previously treated lung cancer? A retrospective study. J Bronchology Interv Pulmonol. 2015;22:11420.

17. Chen F, Miyahara R, Sato T, Sonobe M, Sakai H, Bando T, et al. Usefulness of endobronchial ultrasound in patients with previously treated thoracic malignancy. Interact Cardiovasc Thorac Surg. 2012;14:34-7.

18. Kirita K, Izumo T, Matsumoto Y, Hiraishi Y, Tsuchida T. Bronchoscopic re-biopsy for mutational analysis of non-small cell lung cancer. Lung. 2016;194:371-8.

19. Izumo T, Matsumoto Y, Chavez C, Tsuchida T. Re-biopsy by endobronchial ultrasound procedures for mutation analysis of non-small cell lung cancer after EGFR tyrosine kinase inhibitor treatment. BMC Pulm Med. 2016;16:106.

20. Kang HJ, Hwangbo B, Lee JS, Kim MS, Lee JM, Lee GK. Comparison of epidermal growth factor receptor mutations between metastatic lymph node diagnosed by EBUS-TBNA and primary tumor in non-small cell lung cancer. PLoS One. 2016;11:e0163652.

21. Rusch VW, Asamura H, Watanabe H, Giroux DJ, Rami-Porta $\mathrm{R}$, Goldstraw $\mathrm{P}$, et al. The IASLC lung cancer staging project: a proposal for a new international lymph node map in the forthcoming seventh edition of the TNM classification for lung cancer. J Thorac Oncol. 2009;4:568-77.

22. Kadokura M. Bronchial deformation associated with lobectomy. Ann Thorac Cardiovasc Surg. 2015;21:301-4.

23. Ueda K, Tanaka T, Hayashi M, Tanaka N, Li TS, Hamano K. Right middle lobe transposition after upper lobectomy: influence on postoperative pulmonary function. Thorac Cardiovasc Surg. 2013;61:138-43.

24. Karpathiou G, Giatromanolaki A, Koukourakis MI, Mihailidis V, Sivridis E, Bouros D, et al. Histological changes after radiation therapy in patients with lung cancer: a prospective study. Anticancer Res. 2014;34:3119-24.

25. Choi YW, Munden RF, Erasmus JJ, Park KJ, Chung WK, Jeon SC, et al. Effects of radiation therapy on the lung: radiologic appearances and differential diagnosis. Radiographics. 2004; 24:985-97.

26. Leo F, Pelosi G, Sonzogni A, Chilosi M, Bonomo G, Spaggiari 
L. Structural lung damage after chemotherapy fact or fiction? Lung Cancer. 2010;67:306-10.

27. Szlubowski A, Herth FJ, Soja J, Kolodziej M, Figura J, Cmiel A, et al. Endobronchial ultrasound-guided needle aspiration in non-small-cell lung cancer restaging verified by the transcervical bilateral extended mediastinal lymphadenectomy-a prospective study. Eur J Cardiothorac Surg. 2010;37:1180-4.

28. Herth FJ, Annema JT, Eberhardt R, Yasufuku K, Ernst A, Krasnik $\mathrm{M}$, et al. Endobronchial ultrasound with transbronchial needle aspiration for restaging the mediastinum in lung cancer. J Clin Oncol. 2008;26:3346-50.

29. Yarmus L, Akulian J, Gilbert C, Feller-Kopman D, Lee HJ, Zarogoulidis $\mathrm{P}$, et al. Optimizing endobronchial ultrasound for molecular analysis: how many passes are needed? Ann Am Thorac Soc. 2013;10:636-43.

30. Yu HA, Arcila ME, Rekhtman N, Sima CS, Zakowski MF, Pao $\mathrm{W}$, et al. Analysis of tumor specimens at the time of acquired resistance to EGFR-TKI therapy in 155 patients with EGFRmutant lung cancers. Clin Cancer Res. 2013;19:2240-7.

31. Sequist LV, Waltman BA, Dias-Santagata D, Digumarthy S, Turke AB, Fidias P, et al. Genotypic and histological evolution of lung cancers acquiring resistance to EGFR inhibitors. Sci Transl Med. 2011;3:75ra26.
32. Kuiper JL, Heideman DA, Thunnissen E, Paul MA, van Wijk AW, Postmus PE, et al. Incidence of T790M mutation in (sequential) rebiopsies in EGFR-mutated NSCLC-patients. Lung Cancer. 2014;85:19-24.

33. Oxnard GR, Arcila ME, Sima CS, Riely GJ, Chmielecki J, Kris $\mathrm{MG}$, et al. Acquired resistance to EGFR tyrosine kinase inhibitors in EGFR-mutant lung cancer: distinct natural history of patients with tumors harboring the T790M mutation. Clin Cancer Res. 2011;17:1616-22.

34. Wang F, Fang P, Hou DY, Leng ZJ, Cao LJ. Comparison of epidermal growth factor receptor mutations between primary tumors and lymph nodes in non-small cell lung cancer: a review and meta-analysis of published data. Asian Pac J Cancer Prev. 2014;15:4493-7.

35. Hata A, Katakami N, Yoshioka H, Kaji R, Masago K, Fujita S, et al. Spatiotemporal T790M heterogeneity in individual patients with EGFR-mutant non-small-cell lung cancer after acquired resistance to EGFR-TKI. J Thorac Oncol. 2015;10: 1553-9.

36. Hwangbo B, Lee HS, Lee GK, Lim KY, Lee SH, Kim HY, et al. Transoesophageal needle aspiration using a convex probe ultrasonic bronchoscope. Respirology. 2009;14:843-9. 